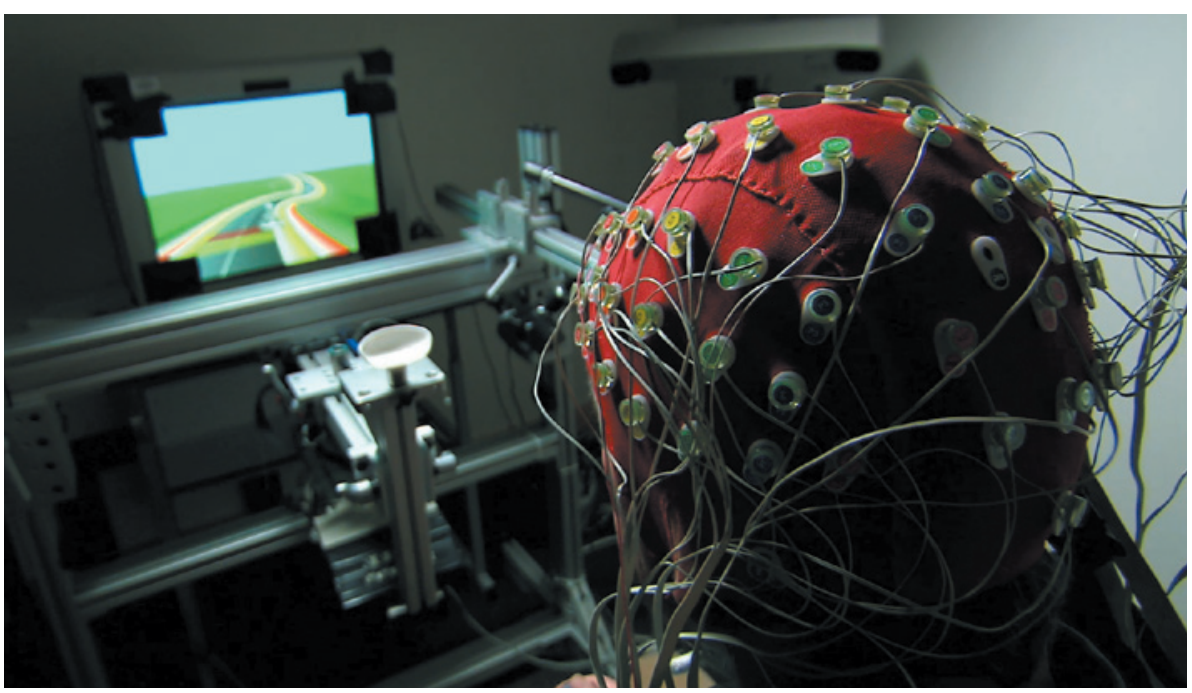

Improved mental skills that come from playing a video game are mirrored by increased brain activity.

NEUROSCIENCE

\title{
Gaming improves multitasking skills
}

\section{Study reveals plasticity in age-related cognitive decline.}

\section{BY ALISON ABBOTT}

S ixty-five-year-old Ann Linsey was starting to worry about how easily she got distracted from whatever she was doing. "As you get older, it seems harder to do more things at once," she says. Then she enrolled in a study to test whether playing a game could improve fading cognitive skills in older people - and was impressed by what it did for her. "I was frustrated because I felt I was losing my faculties. Now I’ve learnt how to focus my attention."

Commercial companies have claimed for years that computer games can make the user smarter, but have been criticized for failing to show that improved skills in the game translate into better performance in daily life ${ }^{1}$. Now a study published this week in Nature ${ }^{2}$ - the one in which Linsey participated - convincingly shows that if a game is tailored to a precise cognitive deficit, in this case multitasking in older people, it can indeed be effective.

Led by neuroscientist Adam Gazzaley of the University of California, San Francisco, the study found that a game called NeuroRacer can help older people to improve their capacity to multitask - and the effect seems to carry over to tasks in everyday life and is still there after six months. The study also shows how patterns of brain activity change as those cognitive skills improve.
NeuroRacer is a three-dimensional video game in which players steer a car along a winding, hilly road with their left thumb, while keeping an eye out for signs that randomly pop up. If the sign is a particular shape and colour, players have to shoot it down using a finger on their right hand. This multitasking exercise, says Gazzaley, draws on a mix of cognitive skills just as real life does - such as attention focusing, task switching and working memory (the ability to temporarily hold multiple pieces of information in the mind).

Gazzaley and his colleagues first recruited around 30 participants for each of six decades of life, from the 20 s to the 70 s, and confirmed that multitasking skills as measured by the game deteriorated linearly with age. They then recruited 46 participants aged $60-85$ and put them through a 4 -week training period with a version of NeuroRacer that increased in difficulty as the player improved.

After training, subjects had improved so much that they achieved higher scores than untrained 20-year-olds, and the skill remained six months later without practice.

The scientists also conducted a battery of cognitive tests on the participants before and after training. Certain cognitive abilities that were not specifically targeted by the game improved and remained improved - such as working memory and sustained attention. Both skills are important for daily tasks, from reading a newspaper to cooking a meal.

That is significant, says Gazzaley. "NeuroRacer doesn't demand too much of those particular abilities - so it appears that the multitasking challenge may put pressure on the entire cognitive control system, raising the level of all of its components."

The team also recorded brain activity using electroencephalography while participants played NeuroRacer. As their skills increased, so did activity in the prefrontal cortex of the brain, which is associated with cognitive control, in a manner that correlated with improvements in sustained-attention tasks. Activity also increased in a neural network linking the prefrontal cortex with the back of the brain.

The industry that has grown around selling brain-training computer games has polarized opinion about the effectiveness of brain-training packages, says cognitive neuroscientist Torkel Klingberg of the Karolinska Institute in Stockholm. "Some companies are not based on real science, and have made unrealistic claims," he says. "On the other hand, some psychologists have claimed that working memory and attention are fixed and can't be trained up."

But Gazzaley's study confirms that cognitive function can be improved - if you design training methods properly, says Klingberg, who is a consultant for Cogmed, a company he founded in 1999 to market computer-based training methods, particularly for people with attention-deficit disorders ${ }^{3}$.

Last year, Gazzaley also co-founded a company, called Akili, for which he is an adviser. It is developing a commercial product similar to NeuroRacer, which remains a research tool, and will seek approval from the US Food and Drug Administration to market it as a therapeutic agent. A 'games' approach might also help people with particular cognitive deficits, such as depression or schizophrenia, adds Daphne Bavelier, a cognitive neuroscientist at the University of Geneva in Switzerland, who develops computer games to improve brain function and who also advises Akili.

Gazzaley cautions against over-hyping: "Video games shouldn't now be seen as a guaranteed panacea." But Linsey, for her part, is happy with what the game did for her and about her own contribution. "It's been exciting to discover the older brain can learn - and I'm glad my own brain helped make the discovery." -

1. Owen, A. M. et al. Nature 465, 775-778 (2010).

2. Anguera, J. A. et al. Nature 501, 97-101 (2013).

3. Klingberg, T. Trends Cogn. Sci. 14, 317-324 (2010).

\section{CORRECTION}

The News Feature 'Egg engineers' (Nature $\mathbf{5 0 0}, 392-394 ; 2013$ ) wrongly said that second-generation primordial germ cells (PGCs) are often not normal — they usually are. It is first-generation PGCs that often display abnormalities. 